\title{
Shadow Chasing : A Resource Allocation Scheme For Heterogeneous Networks
}

\author{
Ahmed R. Elsherif, Zhi Ding, Xin Liu, Jyri Hämäläinen, and Risto Wichman \\ University of California, Davis, California 95616 \\ \{arelsherif,zding,xinliu\}@ucdavis.edu \\ Aalto University, Aalto, Finland \\ \{jyri.hamalainen, risto.wichman\}@aalto.fi
}

\begin{abstract}
In this paper $^{1}$, we propose a resource allocation scheme for interference mitigation in heterogeneous networks to simultaneously serve both Macrocell User Equipments (MUEs) and Home User Equipments (HUEs). In the proposed Shadow Chasing scheme, a Home eNB (HeNB) uses Downlink Control Information (DCI) together with ACK/NAK feedback to assign its HUEs the Physical Resource Blocks (PRBs) that are also potentially used by outdoor MUEs. Since the HeNB receives an outdated DCI due to backhaul delay, our Shadow Chasing algorithm (SCA) determines a likelihood metric for each PRB being either vacant or assigned to an outdoor MUE. By dynamically separating MUE and HUE PRB assignments, SCA can control the downlink interference to MUE. Our numerical examples demonstrate effective reduction of probability for PRB assignment collision and MUE interference over random assignment and a DCI based assignment that does not take into account the delay effect nor the ACK/NAK signal.
\end{abstract}

\section{INTRODUCTION}

Recent studies have shown that indoor usage makes up more than $50 \%$ of voice calls and more than $70 \%$ of wireless data traffic [1]. Ironically, current wireless networks suffer from poor indoor coverage especially for high-speed data services. Moreover, wireless broadband systems often use high carrier frequencies which suffer heavier attenuation losses that degrade signal quality and decrease data rates. One recent proposal for improving indoor wireless coverage is the promising concept of Heterogeneous Networking (HetNet) which specifically includes the deployment of femtocells [2] for improved indoor coverage.

A femtocell is an indoor base station that connects subscribers at a high speed and low power by reusing the same spectrum occupied by an existing cellular macrocell eNB. Femtocell basestation (FBS) connects

\footnotetext{
${ }^{1}$ This material is based on works supported by the National Science Foundation under Grants 0917251 and 1147930 and a research gift from Fujitsu Inc.
}

with core service network via land connections such as Digital Subscriber Line (DSL), or cable modem as a backhaul channel. Femtocells improve indoor signal quality and reduce co-channel interference by using very low power. From the network operator's point of view, femtocells improve indoor coverage and offload traffic from the macrocell, which helps improve the macrocell throughput and link reliability. Another attractive feature of femtocells is that the cost of equipment and deployment is much lower than that of a macrocell base station deployed by the operator.

The concept of heterogeneous networking, and femtocells in particular, has been already proposed in the standardization process for next generation communication systems such as LTE, LTE-A, and WiMAX [3]. Femtocells are covered by Home eNB (HeNB) in LTE standardization. The main challenge of femtocell-macrocell deployments is interference management due to spectrum sharing where both femtocells and macrocells may share multiple time-frequency physical resources. Indeed, because of control signal delays of backhaul connection and decentralized HeNB resource allocation, both femtocells and macrocells may share multiple timefrequency physical resource blocks (PRBs).

HetNet is a natural evolution from the rigid cell coverage to a more flexible and better radio coverage. Cooperative HetNet focuses on resource allocation and channel access of multiple networks simultaneously to achieve maximum throughput and high spectrum utility. It can be viewed as a more general and intelligent form of cognitive networking. The concept of cognitive radio typically refers to radio units having the ability to sense the environment and to adapt its frequency, power, and transmission schemes. This subject has attracted much research interest (e.g., see [4] for an overview).

In the cooperative HetNet context, the Macrocell User Equipment (MUE) may be viewed as the Primary User (PU) that desires rate assurance, whereas the Home User Equipment (HUE) acts as the Secondary User (SU) in 
cognitive radio scenarios. Substantial works exist in this context, especially on dynamic access strategies that are based on sensing outcomes to solve collision problems. In [5] the primary user is not aware of the secondary user activity. SU can access any available channel but must avoid accessing any channel occupied by the PU. The problem was solved and characterized using a 2D Markov chain framework. New performance metrics were obtained to quantify SU performance such as SU blocking probability, SU interruption probability, SU forced termination probability, and SU non-completion probability.

In [6], nearby SUs would cooperate and sense primary channels. The goal is to maximize SUs total rewards given a certain interference constraint on the PU channel. The SUs know approximately the statistics of primary channel activity and a central controller is used to observe a channel and make access decisions. Several works utilize feedback information to improve SU performance. In [7], the SU uses ACK/NAK response from the PU receivers to estimate the PU status. If a NAK is received, SU applies a backoff and then retransmits again until it receives an ACK. In [8], an SU actively transmits probing signals to observe the changing transmission power of PU in response. In [9], a wideband OFDM cognitive radio dynamically changes its subcarrier usage based on the reactive behaviors (e.g., average power and transmission probability) of narrow-band PU devices.

Femtocell resource management also received recent attention (See [10] for a survey). Several mechanisms have been considered, including downlink power control [11], MIMO beamforming through precode matrix index selection [12], and interference avoidance by overhearing MUE resource block allocation information [13] and by using measurement/distance-based power control [14]. Femtocell interference management can be considered as a special case of HetNet resource management. In [15], the authors propose an adaptation technique that combines null steering of antenna array and spectral band selection in the context of cognitive radio networks. The application of this idea in femtocells for interference control is presented in [16]. The work in [17] addresses the interference problem between two HeNBs.

In [18], the authors proposed an adaptive power control technique to limit the transmission power of femtocells in order to maximize frame utilization. This scheme is, however, oblivious to the QoS need of MUEs that experience HeNB interference. In [19], the authors considered probabilistic assignment of PRBs by the HeNB for uplink. The proposed PRB assignment provides HUE with higher access probability to those PRBs occupied by outdoor MUEs. Such schemes require HeNBs to acquire MBS control signals in a timely manner. Furthermore, both HeNB and MBS need to correctly classify MUEs as indoor versus outdoor user equipments. Additionally, extension of this approach to cases involving multiple femtocells remains an open issue.

In this work, we move beyond the basic cognitive paradigm of "sense before transmit" or "listen-beforetalk" (LBT), which relies only on spectral sensing to avoid collision with primary transmissions. Our work in HetNet is based on a more advanced framework that facilitates HetNet cooperation by controlling secondary networks according to a variety of data link control information from primary users. Such feedback mechanisms are available in most recent cellular systems such as LTE and WiMAX. Exploiting feedback information has some advantages over the more traditional LBT techniques for cognitive radios. One advantage is that it overcomes the hidden receiver node problem. Moreover, it provides a direct indication for the cumulative interference at the MUE, thereby enabling the HUEs to exploit the spare capacity in the system. Using feedbacks in the femtocells context is practical as they are now part of the same operator, which makes it easy to access the macrocell information.

The rest of the paper is organized as follows: In section II, we explain the basic idea of the proposed Shadow Chasing method. The detailed derivation of the proposed method are presented in section III. In section IV, we provide numerical performance comparison of the proposed scheme, a random scheduling scheme, and a DCI-following scheme that does not integrate the delay effect nor the ACK/NAK feedback. Finally, section V concludes this work.

\section{PROPOSED IDEA}

If an HeNB knows that an MUE is far away (outdoor MUE), and also knows which PRBs are assigned to that MUE's downlink, then it can assign the same PRBs to its HUEs with confidence of low downlink interference. The HeNB can classify MUEs as outdoor/indoor using Downlink Control Information (DCI) together with the over-heard ACK/NAK in MUEs uplink feedback. From the DCI, the HeNB learns the PRB assignment of different User Equipments (UEs). If the DCI shows that some PRBs are assigned to a certain MUE but neither ACK nor NAK is heard from that MUE, this means that this MUE is far away enough such that we can safely consider it as an outdoor MUE. On the other hand, if 
either ACK or NAK is heard from an MUE, then the MUE is likely to be nearby since the HeNB can hear its feedback signals. Moreover, the HeNB can learn from the DCI whether some PRBs are empty (not assigned to any MUE).

Based on which MUE is outdoor and whether empty PRBs exist or not, the HeNB can either use the empty PRBs, or use those PRBs assigned to outdoor MUEs without causing considerable interference. However, the main challenge is that DCI may only be acquired by HeNBs through backhaul connection or by overhearing the DCI from the MBS. Hence, the MUE PRB assignment information at the HeNB is outdated. To tackle this problem, we let the HeNB estimate the likelihood that a certain PRB is empty or allocated to an outdoor MUE. Because the HeNB is trying to find and use the PRBs of an outdoor MUE, and because its DCI is delayed, we call our proposed algorithm 'shadow chasing'.

By forming a likelihood table for each PRB, the HeNB assigns its HUEs the PRBs with the largest likelihood metric. For HeNB assignment to cause little interference to neighboring MUEs, the HeNB should first use the PRBs that are most likely to be empty. Next it should use the most likely outdoor PRBs. PRBs that are likely assigned to indoor MUEs would be the least favorable. To reduce the possibility of re-using PRBs of indoor MUEs, the HeNB can compare the likelihood metric with a threshold such that if the likelihood is lower than that threshold for some PRBs, the HeNB does not assign those PRBs. In this case, the HeNB causes less interference to indoor MUEs by lowering HUEs' rates.

\section{Shadow Chasing Algorithm}

To estimate the likelihood metric, we use the outdated DCI together with ACK/NAK information. Instead of merely using the instantaneous ACK/NAK signal, we can also use historical ACK/NAK signal for better results. We heuristically propose the following expression for the likelihood metric of PRB $k$ at time instant $n$,

$L(k, n)=a(k, n) e^{-D / c T_{m}}\left[\gamma b_{i}(k, n)+(1-\gamma) b_{h}(k, n)\right]$.

The time index $n$ is in terms of Transmission Time Interval (TTI) which is one subframe in LTE. The variable $a(k, n)$ is a weighting for the likelihood of PRB $k$ to distinguish between empty and used PRBs. It can be defined as follows

$$
a(k, n)= \begin{cases}a_{\text {empty }} & \text { PRB } k \text { is empty at time } n \\ a_{\text {used }} & \text { otherwise. }\end{cases}
$$

Note that $a_{\text {empty }}$ and $a_{\text {used }}$ are two constants with $a_{\text {empty }} \geq a_{\text {used }}$. The factor $e^{-D / c T_{m}}$ indicates the confidence in the outdated DCI, where $D$ is the delay of the backhaul connection in TTI units. $T_{m}$ is the MBS scheduling period in TTI units and we assume that $T_{m} \geq D$. The constant $c$ determines how aggressive we wish to model the effect of delayed DCI. The choice of the confidence factor to be $e^{-D / c T_{m}}$ is intuitive because the larger the MUE scheduling period $T_{m}$ is relative to the backhaul delay $D$, the more confidence the HeNB has in its outdated DCI information it receives. On the other hand, if $T_{m}$ is comparable to $D$, the actual MUE PRB assignment might be completely different from the received outdated DCI. This leads to more incorrect HeNB assignment decisions.

The term between square brackets in (1) describes the likelihood as a function of the ACK/NAK signal. The ACK/NAK signal bit indicates the success/failure of packet reception and decoding. Unlike DCI or CQI, ACK/NAK information is not on the PRB level. However, using the DCI we can find the PRBs assigned to a certain MUE and, therefore, update the likelihood metric according to ACK/NAK for the whole corresponding PRBs. The variable $b_{i}(k, n)$ is defined as:

$$
b_{i}(k, n)= \begin{cases}b_{d h} & \text { ACK/NAK signal is not heard } \\ b_{h a} & \text { HeNB hears an ACK } \\ b_{h n} & \text { HeNB hears a NAK }\end{cases}
$$

where $b_{d h} \geq b_{h a} \geq b_{h n}$.

If the HeNB is unable to hear the ACK/NAK signal from a specific MUE, it likely means that it is an outdoor user. Thus, all assigned PRBs of that MUE should have higher likelihood metric. If the HeNB hears an ACK from an MUE, it means it is likely close (indoor) but is experiencing good channel conditions. If, instead, the HeNB hears a NAK, then the MUE is likely indoor and is already experiencing a poor channel condition. Hence, $b_{h n}$ should be small to avoid reusing the PRBs of that MUE. The variable $b_{h}(k, n)$ depends on the history of the ACK/NAK signal. We can either store the ACK/NAK signals themselves or store the mapping $b_{i}(k, n)$. This way, $b_{i}(k, n)$ refers to the instantaneous value, whereas $b_{h}(k, n)$ refers to its long term average. The value of $b_{h}(k, n)$ can be obtained from the past instantaneous values $b_{i}(k, n)$ through simple movingwindow averaging as

$$
b_{h}(k, n)=\frac{1}{N_{b}} \sum_{m=n-N_{b}}^{n-1} b_{i}(k, m)
$$


where $N_{b}$ is the length of the moving average window for $b_{i}(k, m)$. Finally, $\gamma$ is a forgetting factor controlling how much we rely on the instantaneous value versus the history of ACK/NAK information. The HeNB updates the likelihood table each time instant $n$ (i.e. each TTI) and makes the scheduling decision accordingly each $T_{f}$ TTIs. Fig. 1 illustrates the timing diagram of the MBS/HeNB scheduling together with the reception of DCI information.

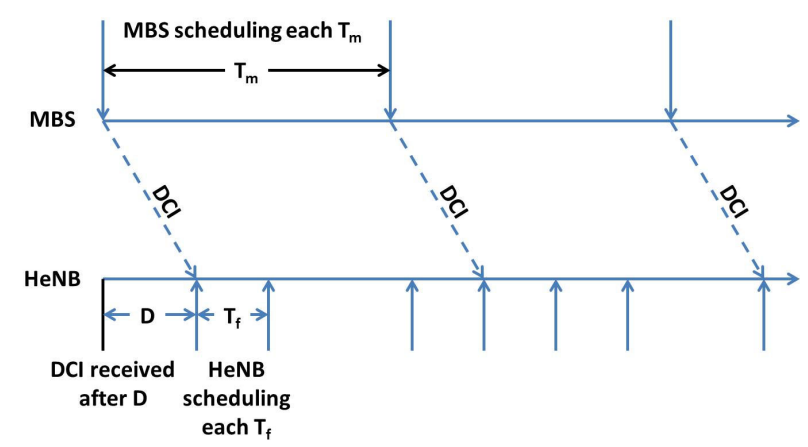

Fig. 1: Illustration of the Shadow Chasing algorithm.

The HeNB calculates the likelihood for each PRB and assigns the PRBs that have the largest likelihood metric as described in Section II. If the likelihood metric is lower than a threshold $\alpha$ for a certain PRB, the HeNB avoids using this PRB in scheduling to lower the possibility of severe interference to nearby MUEs. The threshold $\alpha$ controls how aggressive the HeNB schedules a PRB. A large value of $\alpha$ means conservative HeNB scheduling such that the HeNB assigns less PRBs to its HUEs to avoid scheduling on PRBS that may be assigned to nearby indoor MUEs.

\section{Simulation RESUlts}

We present a numerical simulation test of a HetNet that utilizes the shadow chasing algorithm (SCA). We consider a HetNet with $N_{m}$ MUEs and one HeNB. Each MUE has a certain PRB requirement and the HeNB has a desired number of PRBs to satisfy the requirements of its associated HUEs. For mandatory spectrum sharing, we consider the total number of required PRBs for all MUEs and HUEs to be greater than the maximum number of available PRBs. The distance between the MUE $i$ and its serving basestation is denoted by $d_{m}(i)$, and its distance from the HeNB is $d_{h}(i)$. The distance $d_{h}(i)$ for all MUEs are chosen so that some of the MUEs are outdoor (i.e. $d_{h}(i)$ is large enough). The simulation parameters are given in Table I.

\begin{tabular}{|c|c|}
\hline Parameter & Value \\
\hline Outdoor MUEs PRBs & $36.36 \%$ \\
\hline Indoor MUEs PRBs & $54.55 \%$ \\
\hline Empty PRBs & $9.09 \%$ \\
\hline HUE requested PRBs & $45.45 \%$ \\
\hline MBS, HeNB Tx Powers & $47 \mathrm{dBm}, 20 \mathrm{dBm}$ \\
\hline MBS to MUE Path Loss & $151.1+42.8 \cdot \log 10\left(d_{m}(i) / 1000\right)$ \\
\hline HeNB to HUE Path Loss & $\begin{array}{c}127+30 \cdot \log 10\left(d_{f} / 1000\right) \\
d_{f}: \text { distance between HeNB } \\
\text { and HUE (in meters) }\end{array}$ \\
\hline
\end{tabular}

TABLE I: Simulation Parameters.

As a performance metric, we examine the probability of PRB collision versus the MUE scheduling period $T_{m}$, where the probability of PRB collision is defined as the probability that the HeNB uses a PRB assigned to an indoor MUE. As $D \rightarrow 0$ and if the number of PRBs requested by the HeNB is less than the total number of PRBs requested by outdoor MUEs, our proposed scheme should have a zero probability of collision. However, because the backhaul connection delay, $D$, is typically in the order of tens of TTIs (assuming a DSL backhaul), the probability of collision can be substantial.

For performance comparison, we also consider two additional and more naive methods of PRB assignment: random assignment scheme and DCI-following scheme. In random assignment, the HeNB blindly assigns PRBs without exploiting any DCI information about MUE scheduling. In fact, it does nothing to avoid interference to near-by MUEs. The DCI-following scheme is a smarter but naive scheme that uses the DCI as if it gives the accurate PRB assignment without accounting for the backhaul delay. This scheme can be thought of as a special case of the SCA which assigns PRB according to the DCI as:

$$
L(k, n)=a(k, n) .
$$

Moreover, DCI-following does not exploit ACK/NAK and is unable to distinguish between PRBs assigned to indoor or outdoor MUEs.

Performance evaluation of the three schemes is shown in Fig. 2 for different values of $T_{f}$. In this simulation, the value of $D$ is set to 50 TTI. Moreover, the value of $\alpha$ is set to 0 , which refers to aggressive HeNB scheduling. Intuitively, as $T_{m}$ increases relative to the backhaul delay $D$, the probability of collision decreases. When $T_{m}$ and $D$ are comparable, the effect of $D$ becomes more significant. On the other hand, the probability of collision grows if the HeNB scheduling period $T_{f}$ increases because the HeNB becomes less adaptive to 
MUE feedback signals and scheduling updates. From Fig. 2, it is clear that random assignment gives the largest probability of PRB collision. As expected, the performance of the random assignment scheme is independent of $T_{m}$ and $T_{f}$. Moreover, SCA outperforms the DCI-following scheme for all values of $T_{f}$ since it uses likelihood information for scheduling decisions. Because the ACK/NAK signal is already sent from MUEs to their MBS for each subframe, there is no extra spectral overhead. Thus, the throughput enhancement is obtained at no extra cost

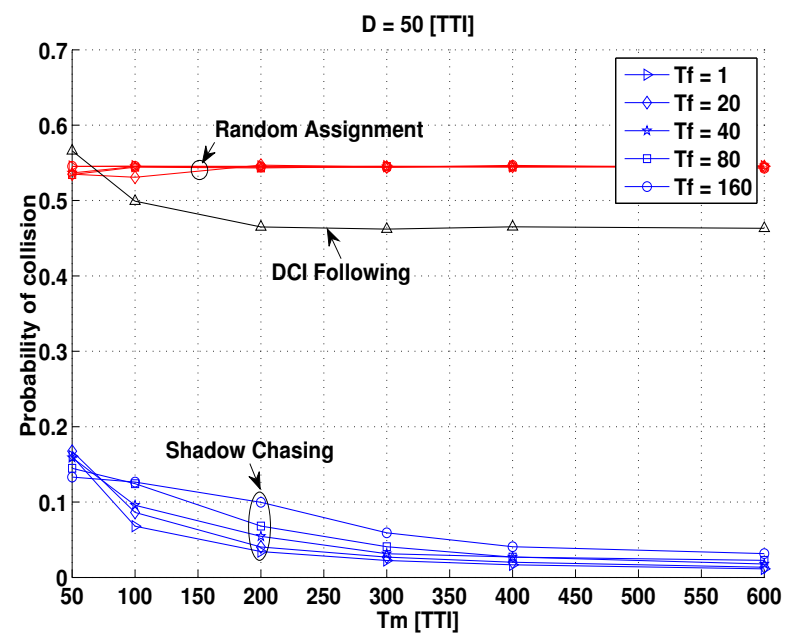

Fig. 2: PRB collision rate vs $T_{m}$ for different $T_{f}$.

Another performance metric we consider is the sum MUE rate. Fig. 3 compares the sum MUE rate for three schemes. The value of $D$ is also set to 50 TTI as in the previous simulation. As seen from Fig. 3, when $T_{m}$ increases relative to $D$, the probability of $\mathrm{HeNB}$ interference to indoor MUEs decreases. This in turn will drop the interference experienced by indoor MUEs and increase the sum of the MUE rates as in Fig. 3. Because in this simulation $\alpha=0$, HeNB scheduling is aggressive in the sense that the HUE gets all the requested PRBs. It is thus clear that the HUE rate, in this case, is independent of the MUE scheduling period, $T_{m}$, and the HUE scheduling period, $T_{f}$.

Another way to compare the performance of SCA with DCI-following is to fix a value for $T_{m}$ and determine the probability of collision versus $D$. In Fig. 4, we fix $T_{m}=300$ and provide the probability of collision for different values of $T_{f}$. As $D$ increases, the probability of PRB collision becomes higher for both shadow chasing and DCI-following. However, shadow chasing still offers substantial gain over DCI-following. It is worth noting

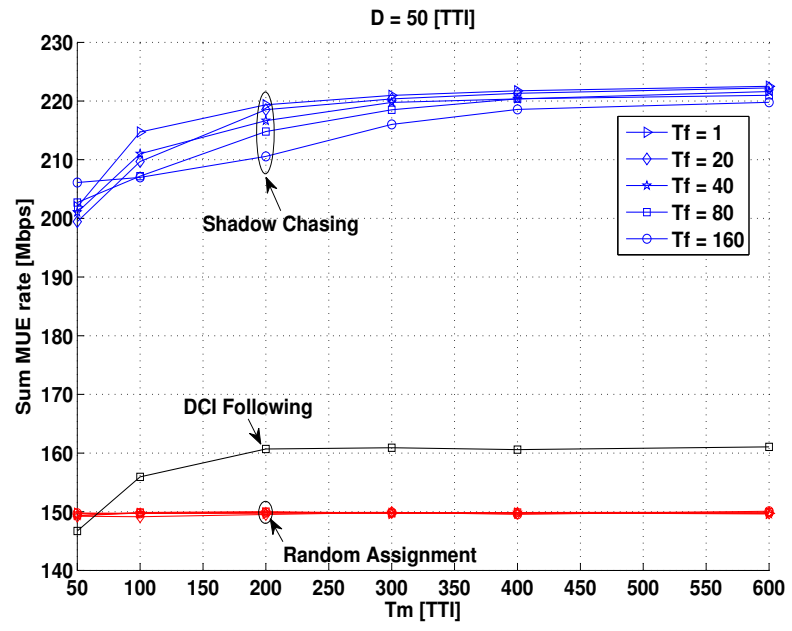

Fig. 3: Sum of MUEs rates comparison.

that when $D$ is closer to $T_{m}$, DCI-following will be using incorrect (misleading) DCI. Therefore, the HeNB might schedule a large number of indoor PRBs, thereby leading to a higher probability of PRB collision.

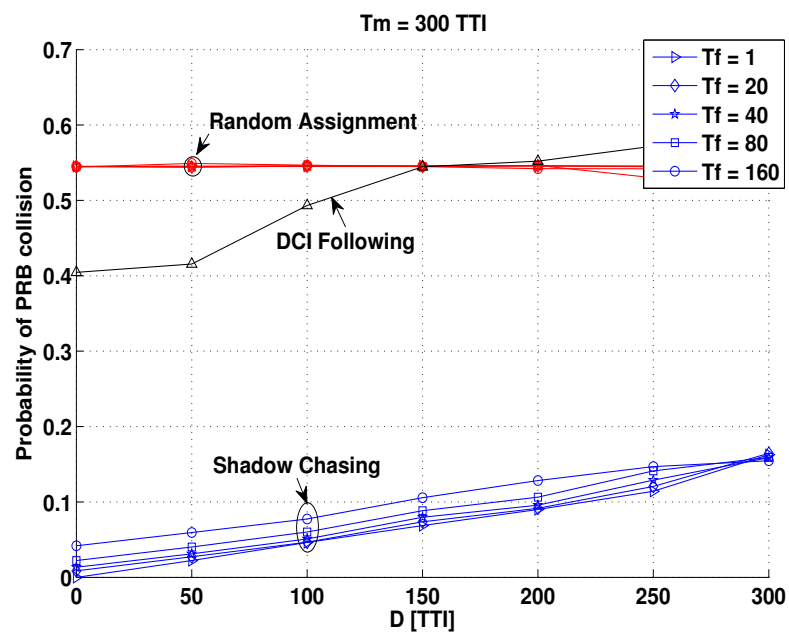

Fig. 4: PRB collision rate vs $D$ for different $T_{f}$.

To examine the effect of the threshold parameter $\alpha$ on performance, we find the data rates for various values of $\alpha$ in Fig. 5. As the value of $\alpha$ increases, the HeNB becomes more conservative and the probability of collision decreases. Consequently, the sum MUE rate grows. At the same time, the HUE rate decreases as it is now getting fewer PRBs than requested. For $\alpha=0.6$ and at $T_{m}=50$, some PRBs are prohibited for the HUE because their likelihood metric is below 0.6. Hence, the HUE rate decreases. For $\alpha=0.7$, all the PRBs are prohibited for the HUE and, as a result, the HUE has zero 
rate and the sum MUE rate increases significantly. By making $\alpha$ higher, we observe that, for $\alpha=0.8$, the HUE receives no rate up to $T_{m}=100$. Beyond that point, it then receives some PRBs as $T_{m}$ grows and the likelihood metric increases. We further show the total rate which is the sum of all rates from HUE and MUEs. From these results, we see that $\alpha$ affects the total rate of the heterogeneous network by determining how conservative or aggressive the HeNB PRB assignment is.

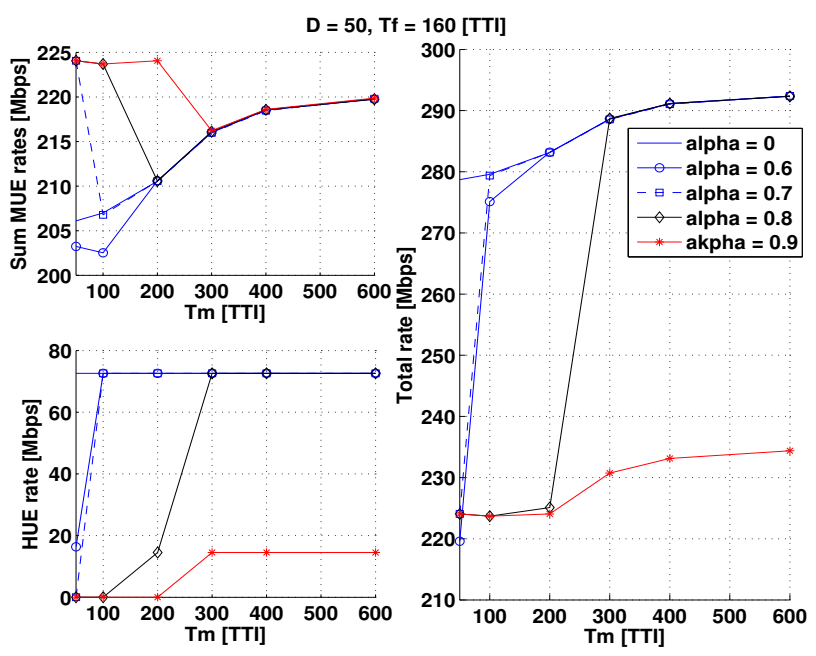

Fig. 5: Performance metrics with different $\alpha$.

\section{CONCLUSION}

We proposed a simple and novel technique for resource allocation in HetNet known as shadow chasing. In the proposed algorithm, the HeNB receives DCI from the MBS through backhaul and uses it jointly with its over-heard ACK/NAK signal to classify neighboring MUEs into outdoor or indoor probabilistically. Using a likelihood table for each PRB, the HeNB can assign its users those PRBs that are the most likely to be empty or used by outdoor MUEs. If outdoor MUEs are far away, they would experience less interferences. Even if these outdoor MUEs are not very far away and may suffer from HUE interference, the over-heard ACK/NAK signals from those MUEs will allow the HeNB to reduce the likelihood metric of assigning their corresponding PRBs so as to mitigate interference in subsequent TTIs. We provide numerical examples that illustrate the effective reduction of packet collision and the spectrum efficiency achieved by the shadow-chasing allocation algorithm.

\section{REFERENCES}

[1] Presentations by ABI Research, Picochip, Arivana, IP access, Gartner, Telefonica Espana, 2nd Int'l Conf. Home Access Points and Femtocells.
[2] V. Chandrasekhar, J. Andrews, and A. Gatherer, "Femtocell Networks: A Survey," IEEE Communications Magazine, vol. 46, no. 9, pp. 59-67,Sept. 2008.

[3] J. Zhang and G. de la Roche, Femtocells : Technologies and Deployment, JohnWiley \& Sons, New York, NY, USA, 2010.

[4] I. F. Akyildiz, W.-Y. Lee, M. C. Vuran, and S. Mohanty, "Next Generation/Dynamic Spectrum Access/Cognitive Radio Wireless Networks: a Survey," The International Journal of Computer and Telecommunications Networking, 50(13):2127-2159, 2006.

[5] Y. Zhang, "Dynamic Spectrum Access in Cognitive Radio Wireless Networks," in IEEE International Conference on Communications, Dec. 2008 : 4927-4932.

[6] J. Unnikrishnan, and V. V. Veeravalli, "Algorithms for Dynamic Spectrum Access with Learning for Cognitive Radio.” IEEE Transactions on Signal Processing 58(2):750-760, 2008.

[7] Q. Zhao, L. Tong, A. Swami and Y. Chen, "Decentralized cognitive MAC for opportunistic spectrum access in ad hoc networks: A POMDP framework," IEEE Journal on Selected Areas in Communications 25.3 (2007) : 589-600.

[8] R. Zhang and Y. C. Liang, "Exploiting Hidden Power Feedbacks in Cognitive Radio Networks," 3rd IEEE Intl. Symp. on New Frontiers in Dynamic Spectrum Access Networks, 2008.

[9] H. Rahul, N. Kushman, D. Katabi, C. Sodini, and F. Edalat, "Learning to Share: Narrowband-Friendly Wideband Networks," SIGCOMM Comput. Commun. Rev., 38(4):147-158, 2008.

[10] V. Chandrasekhar, J. Andrews, and A. Gatherer, "Femtocell Networks: a Survey," IEEE Communications Magazine, 46(9):59-67, 2008.

[11] X. Li, L. Qian, and D. Kataria, "Downlink Power Control in Co-channel Macrocell Femtocell Overlay," 43rd Conference on Information Sciences and Systems, pages 383-388, 2009.

[12] C. Jiang, L. J. Cimini, and N. Himayat, "MIMO Mode Adaptation in Femtocellular Systems," Computer Engineering, pages 122-127, 2011.

[13] M. Sahin, I. Guvenc, M. Jeong, and H. Arslan, "Handling CCI and ICI in OFDMA Femtocell Networks Through Frequency Scheduling," IEEE Trans. on Consumer Electronics, 55(4):19361944, 2009.

[14] H. Claussen, F. Pivit, and L. T. W. Ho, "Self-Optimization of Femtocell Coverage to Minimize the Increase in Core Network Mobility Signaling," Bell Labs Technical Journal, 14(2):155183, 2009.

[15] J. Mitola, "Cognitive radio for flexible mobile multimedia communications," in IEEE International Workshop on Mobile Multimedia Communications (MoMuC99) (Cat. No.99EX384), vol. 22102, pp. 3-10, 1999.

[16] M. Husso, J. Hämäläinen, R. Jantti, and A. M. Wyglinski, "Adaptive Antennas and Dynamic Spectrum Management for Femtocellular Networks: A Case Study," in 3rd IEEE Symposium on New Frontiers in Dynamic Spectrum Access Networks, pp. 15, Oct. 2008.

[17] M. Husso, Z. Zheng, J. Hämäläinen and E. Mutafungwa, "Dominant interferer mitigation in closed femtocell deployment," in IEEE 21st Intl. Symp. on Personal Indoor and Mobile Radio Communications, pp.169-174, 2010.

[18] P. Mach, Z. Becvar, "Dynamic Power Control Mechanism for Femtocells Based on the Frame Utilization," in 6th International Conference Wireless and Mobile Communications, 2010.

[19] Z. Zheng, J. Hämäläinen, and Y. Yang, "On Uplink Power Control Optimization and Distributed Resource Allocation in Femtocell Networks," in VTC Spring 2011 BeFEMTO workshop, 15 May, (Budapest, Hungary), 2011. 